\title{
Reconstruction of the Sturm-Liouville Operator on a Graph from Subinterval Nodes
}

\author{
Chuan-Fu Yang* and Sheng-Yu Guan
}

\begin{abstract}
We consider inverse subinterval-nodal problems for the Sturm-Liouville operator on a star graph with mixed boundary conditions in pendant vertices and the standard matching conditions at the interior vertex. Can subinterval nodes recognize star-shaped quantum graphs? In this paper we give a positive answer. It is shown that the data of subinterval-nodes near the interior vertex can uniquely determine the potential on a graph and boundary conditions.
\end{abstract}

\section{Introduction}

We consider a compact star graph $G$ with the vertex set $V=\left\{v_{0}, v_{1}, v_{2}, \ldots, v_{m}\right\}$ and edge set $E=\left\{e_{1}, \ldots, e_{m}\right\}$, where $m \geq 3, v_{0}$ is the internal vertex, $v_{1}, \ldots, v_{m}$ are the boundary vertices and each edge is of equal length 1 . For convenience, we parameterize each $e_{j}$ by $x \in[0,1]$ such that $x=0$ corresponds to the boundary vertex $v_{j}$ and $x=1$ corresponds to the internal vertex $v_{0}$. Consider the boundary value problem $L:=L(q, h)$ on the graph $G$ generated by the following Sturm-Liouville equations

$$
-y_{j}^{\prime \prime}+q_{j}(x) y_{j}=\lambda y_{j}, \quad x \in(0,1), j=1,2, \ldots, m,
$$

together with the Robin and/or Dirichlet conditions on the boundary vertices

$$
\begin{aligned}
y_{j}^{\prime}(0, \lambda)-h_{j} y_{j}(0, \lambda)=0, \quad j=1,2, \ldots, p, \\
y_{j}(0, \lambda)=0, \quad j=p+1, p+2, \ldots, m,
\end{aligned}
$$

and the standard matching conditions at the internal vertex

$$
y_{1}(1, \lambda)=y_{j}(1, \lambda), \quad j=2, \ldots, m, \quad \sum_{j=1}^{m} y_{j}^{\prime}(1, \lambda)=0,
$$

Received March 10, 2020; Accepted February 7, 2021.

Communicated by Jenn-Nan Wang.

2020 Mathematics Subject Classification. 34A55, 34B45, 47E05.

Key words and phrases. star graph, Sturm-Liouville operator, subinterval nodes, inverse nodal problem, inverse spectral problems.

This work was supported in part by the National Natural Science Foundation of China (11871031) and by the Natural Science Foundation of the Jiangsu Province of China (BK 20201303).

${ }^{*}$ Corresponding author. 
where $\lambda$ is the spectral parameter, $1 \leq p<m, h=\left\{h_{j}\right\}_{j=1,2, \ldots, p}$ are real constants, the potential $q=\left\{q_{j}\right\}_{j=1,2, \ldots, m}$ is real-valued function in $\bigoplus_{j=1}^{m} L_{2}[0,1]$.

In this work, we consider the inverse nodal problems on a star graph with mixed boundary conditions by using subinterval nodal data, which amounts to subinterval nodes (zeros) of eigenfunctions. These problems are related to some questions in mechanics and mathematical physics (see, for example, [14]). Inverse nodal problems for SturmLiouville operators on an interval have been studied fairly completely in $5,6,9,11,14,19$ and other papers. Differential operators on graphs (networks, trees) often appear in natural sciences and engineering (see $8,10,15,17]$ and the references therein). On inverse nodal problems for differential operators on graphs there are only a few findings. For example, the works $[2,18,20$, prove that the set of nodal points, which is dense in the whole interval considered, uniquely determines the parameters of the boundary conditions and the potential functions on a graph. As well as in the work $\sqrt{3}$ the uniqueness of the potential for given nodal data is proved and authors give a construction of the potential as a limit of a sequence of functions whose $n$th term is dependent only on the $n$th eigenvalue and its associated nodal data, which is dense in the whole interval considered.

When solving the inverse problem, to avoid over-determinedness of the inverse nodal problem one hopes to get the expected result with the least/optimal input data. In the inverse node problem of a finite interval, the node data of subinterval is enough to determine the uniqueness [5,6, 19. Inspired by it, together with the well-known inverse spectral analysis with partial information on the potential and eigenvalues [4,7], can the node data on the subinterval determine the quantum graph? This paper gives a positive answer.

This work establishes uniqueness theorems of the inverse subinterval nodal problems for the Sturm-Liouville operator on a star-type graph. We prove that the data of subintervalnodes near the interior vertex can uniquely determine the potential on a graph and boundary conditions.

An outline of this paper is as follows. In Section 2, some preliminaries are provided. Section 3 deals with the inverse nodal problem on the whole interval. Section 4 is devoted to the statements of the inverse subinterval nodal problems.

\section{Preliminaries}

In this section, we review some facts about the Sturm-Liouville operator $L$ on the graph $G$ and the growth of entire functions. Let $C_{i}(x, \lambda), i=1,2, \ldots, p$ and $S_{i}(x, \lambda), i=$ $p+1, p+2, \ldots, m$ be the solutions of equations (1.1) under the initial conditions

$$
C_{i}(0, \lambda)=1, \quad C_{i}^{\prime}(0, \lambda)=h_{i}, \quad S_{i}(0, \lambda)=0, \quad S_{i}^{\prime}(0, \lambda)=1 .
$$


Then for large $|\lambda| \rightarrow \infty$, the solutions $C_{i}(x, \lambda)$ and $S_{i}(x, \lambda)$ have the asymptotic formulas, uniformly in $x \in[0,1]$,

$$
\begin{aligned}
& C_{i}(x, \lambda)=\cos \rho x+\left(h_{i}+\left[q_{i}\right]_{x}\right) \frac{\sin \rho x}{\rho}+o\left(\frac{e^{\tau x}}{\rho}\right), \\
& S_{i}(x, \lambda)=\frac{\sin \rho x}{\rho}-\left[q_{i}\right]_{x} \frac{\cos \rho x}{\rho^{2}}+o\left(\frac{e^{\tau x}}{\rho^{2}}\right),
\end{aligned}
$$

where $\lambda=\rho^{2}, \tau=|\operatorname{Im} \rho|$, and $\left[q_{i}\right]_{x}:=\frac{1}{2} \int_{0}^{x} q_{i}(t) d t$.

As is shown in [1], $L$ has a countable set of real eigenvalues, which can be enumerated as $\left\{\lambda_{n k}\right\}_{n=1}^{\infty}, k=1,2, \ldots, m$ (counting with their multiplicities), and satisfy the following asymptotic formulae

$$
\begin{array}{ll}
\rho_{n 1}=\rho_{n 1}^{0}+\frac{\omega}{n \pi}+o\left(n^{-1}\right), & \\
\rho_{n 2}=\rho_{n 2}^{0}+\frac{\omega}{n \pi}+o\left(n^{-1}\right), & \\
\rho_{n k}=\left(n-\frac{1}{2}\right) \pi+O\left(n^{-1}\right), \quad k \in J_{3}, \\
\rho_{n k}=n \pi+O\left(n^{-1}\right), \quad k \in J_{4},
\end{array}
$$

where $\lambda_{n k}=\rho_{n k}^{2}, \rho_{n 1}^{0}=(n-1) \pi+\arccos \sqrt{\frac{p}{m}}, \rho_{n 2}^{0}=n \pi-\arccos \sqrt{\frac{p}{m}}$,

$$
\omega=\frac{1}{p} \sum_{j=1}^{p} \omega_{j}+\frac{1}{m-p} \sum_{j=p+1}^{m} \omega_{j}, \quad \omega_{j}= \begin{cases}h_{j}+\left[q_{j}\right]_{1} & \text { if } j=1,2, \ldots, p, \\ {\left[q_{j}\right]_{1}} & \text { if } j=p+1, p+2, \ldots, m,\end{cases}
$$

$J_{3}$ and $J_{4}$ are some fixed sets of indices such that $J_{3} \cup J_{4}=\{3,4, \ldots, m\}, J_{3} \cap J_{4}=\emptyset$, $\left|J_{3}\right|=p-1,\left|J_{4}\right|=m-p-1$. For definiteness, we assume that $3 \in J_{3}$ and $4 \in J_{4}$ if these sets are nonempty.

Let $0 \leq a \leq 1$. Denote by $L_{j}$ the Sturm-Liouville problem on each edge $e_{j}$ for $j=1,2, \ldots, m$ defined by

$$
\begin{aligned}
-y_{j}^{\prime \prime}+q_{j}(x) y_{j} & =\lambda y_{j}, & & x \in(0, a), \\
y_{j}^{\prime}(0, \lambda)-h_{j} y_{j}(0, \lambda) & =y_{j}(a, \lambda)=0, & & j=1,2, \ldots, p
\end{aligned}
$$

and

$$
\begin{aligned}
-y_{j}^{\prime \prime}+q_{j}(x) y_{j} & =\lambda y_{j}, & & x \in(0, a), \\
y_{j}(0, \lambda) & =y_{j}(a, \lambda)=0, & & j=p+1, p+2, \ldots, m .
\end{aligned}
$$

The function $M_{j}(x, \lambda)$ defined by

$$
M_{j}(x, \lambda)= \begin{cases}-\frac{C_{j}^{\prime}(x, \lambda)}{C_{j}(x, \lambda)} & \text { if } j=1,2, \ldots, p, \\ -\frac{S_{j}^{\prime}(x, \lambda)}{S_{j}(x, \lambda)} & \text { if } j=p+1, p+2, \ldots, m\end{cases}
$$


is called the Weyl function of $L_{j}$. According to [4], the following asymptotic formulae hold

$$
M_{j}(x, \lambda)=i \rho+o(1)
$$

uniformly in $x \in[\delta, a]$ for $|\lambda| \rightarrow \infty$ in any sector $\varepsilon<\arg (\lambda)<\pi-\varepsilon$ for $\varepsilon>0$, where $\delta \in(0, a]$. In addition, we need a classical estimate of Levinson in [13] and a PhragménLindelöf-type result in $[12]$.

Lemma 2.1. Let $\left\{z_{n}\right\}_{n \geq 1}$ be a sequence of complex numbers so that

$$
\lim _{n \rightarrow \infty} \frac{n}{z_{n}}=d
$$

holds for some $d \in \mathbb{R}$ and there exists a constant $c>0$ such that

$$
\left|z_{n}-z_{m}\right| \geq c|n-m|
$$

Define

$$
f(z)=\prod_{n=1}^{\infty}\left(1-\frac{z^{2}}{z_{n}^{2}}\right)
$$

If $z_{n}=0$, we substitute $1-z^{2} / z_{n}^{2}$ by $z^{2}$. Then for some $C>0$ there hold

$$
|f(z)| \leq C \mathrm{e}^{\pi d|\operatorname{Im} z|+\varepsilon|z|}, \quad \forall \varepsilon>0 \quad \text { as }|z| \rightarrow \infty
$$

and

$$
\left|\frac{1}{f(z)}\right| \leq C \mathrm{e}^{-\pi d|\operatorname{Im} z|+\varepsilon|z|} \quad \text { if }\left|z-z_{n}\right| \geq \frac{1}{8} c .
$$

Lemma 2.2. Let $f(z)$ be an entire function of zero exponential type, i.e.,

$$
\limsup _{r \rightarrow \infty} \frac{\ln M(r)}{r} \leq 0, \quad \text { where } M(r)=\max _{\varphi}\left\{\left|f\left(r e^{i \varphi}\right)\right|\right\}
$$

If $f(z)$ is bounded along a line, then $f(z)$ is a constant. In particular, $f(z) \rightarrow 0$ when $|z| \rightarrow \infty$ along a line, then $f(z) \equiv 0$.

3. Inverse nodal problem on the whole interval

Put

$$
Y(x, \lambda)=\left\{y_{i}(x, \lambda)\right\}_{i=1,2, \ldots, m}, \quad y_{i}(x, \lambda)= \begin{cases}A_{i}(\lambda) C_{i}(x, \lambda) & \text { if } i=1,2, \ldots, p \\ A_{i}(\lambda) S_{i}(x, \lambda) & \text { if } i=p+1, p+2, \ldots, m .\end{cases}
$$

Then the function $Y(x, \lambda)$ satisfies equations and the boundary conditions. If $\lambda^{*}$ is an eigenvalue of the problem then the function $Y\left(x, \lambda_{0}\right)$ is an eigenfunction. 
Using the asymptotic expression (2.2) of the eigenvalue $\lambda_{n 1}:=\rho_{n 1}^{2}$, when $n \rightarrow \infty$, we obtain the asymptotics for the components of the eigenfunction $Y\left(x, \lambda_{n 1}\right)$, uniformly in $x \in[0,1]:$

$$
C_{i}\left(x, \lambda_{n 1}\right)=\cos \rho_{n 1}^{0} x+\frac{\beta_{i}(x)}{2 \pi(n-1)} \sin \rho_{n 1}^{0} x+o\left(\frac{1}{n}\right), \quad i=1,2, \ldots, p,
$$

where $\beta_{i}(x)=2\left[q_{i}\right]_{x}+2 h_{i}-\omega x$, and for $i=p+1, p+2, \ldots, m-1$,

$$
S_{i}\left(x, \lambda_{n 1}\right)=\frac{\sin \rho_{n 1}^{0} x}{\rho_{n 1}^{0}}+\frac{\gamma_{i}(x)}{2 \pi^{2}(n-1)^{2}} \cos \rho_{n 1}^{0} x+o\left(\frac{1}{n^{2}}\right),
$$

where $\gamma_{i}(x)=-2\left[q_{i}\right]_{x}+\omega x$.

Similarly, taking $\lambda_{n 2}:=\rho_{n 2}^{2}$ in $(2.2)$, for large $n \rightarrow \infty$, we also get the asymptotics for the components of the eigenfunction $Y\left(x, \lambda_{n 2}\right)$, uniformly in $x \in[0,1]$ :

$$
S_{m}\left(x, \lambda_{n 2}\right)=\frac{\sin \rho_{n 2}^{0} x}{\rho_{n 2}^{0}}+\frac{\gamma_{m}(x)}{2 \pi^{2} n^{2}} \cos \rho_{n 2}^{0} x+o\left(\frac{1}{n^{2}}\right) .
$$

Fix $i=1,2, \ldots, m$. There exists $N_{0}$ such that for $n \geq N_{0}$ the function $C_{i}\left(x, \lambda_{n 1}\right)$ (or $\left.S_{i}\left(x, \lambda_{n 1}\right), S_{m}\left(x, \lambda_{n 2}\right)\right)$ has exactly $n-1$ (simple) zeros inside the interval $(0,1)$, that is, $0<x_{n i}^{1}<\cdots<x_{n i}^{n-1}<1$. The point sets $X_{i}:=\left\{x_{n i}^{j}\right\}_{n \geq N_{0}}(i=1,2, \ldots, m-1$, $j=1,2, \ldots, n-1)$ are called the nodes on the edge $e_{i}$ with respect to the eigenvalues $\lambda_{n 1}$, $X_{m}:=\left\{x_{n m}^{j}\right\}_{n \geq N_{0}}$ with respect to the eigenvalues $\lambda_{n 2}$.

Denote

$$
\alpha_{n}^{j}:=\frac{\left(j-\frac{1}{2}\right) \pi}{\rho_{n 1}^{0}}, \quad \beta_{n}^{j}:=\frac{j \pi}{\rho_{n 1}^{0}}, \quad \gamma_{n}^{j}:=\frac{j \pi}{\rho_{n 2}^{0}},
$$

where $j=1,2, \ldots, n-1$. Taking asymptotic formula 2.1 for eigenfunctions into account, for large $n$, the following asymptotic formulae for the nodes hold uniformly in $j$ :

$$
x_{n i}^{j}= \begin{cases}\alpha_{n}^{j}+\frac{\beta_{i}(x)}{2 \pi^{2}(n-1)^{2}}+o\left(\frac{1}{n^{2}}\right) & \text { if } i=1,2, \ldots, p, \\ \beta_{n}^{j}-\frac{\gamma_{i}(x)}{2 \pi^{2}(n-1)^{2}}+o\left(\frac{1}{n^{2}}\right) & \text { if } i=p+1, p+2, \ldots, m-1, \\ \gamma_{n}^{j}-\frac{\gamma_{m}(x)}{2 \pi^{2}(n-1)^{2}}+o\left(\frac{1}{n^{2}}\right) & \text { if } i=m .\end{cases}
$$

Note that for the fixed $i=1,2, \ldots, m$ the nodal set $X_{i}$ is dense in $(0,1)$. Without loss of generality one may assume that $\omega=0$, which can be achieved by shifting a constant $\omega$. Analyzing the asymptotic expressions $x_{n i}^{j}$ we have the following statements.

Fixed $i=1,2, \ldots, m$ and $x \in[0,1]$. Suppose that $X_{i}^{0} \subset X_{i}$ is dense on $(0,1)$ and choose $\left\{x_{n i}^{j_{n i}}\right\} \subset X_{i}^{0}$ such that $\lim _{n \rightarrow \infty} x_{n i}^{j_{n i}}=x$. Then the following finite limits hold:

(a) $\lim _{n \rightarrow \infty} 2 \pi^{2}(n-1)^{2}\left(x_{n i}^{j_{n i}}-\alpha_{n}^{j}\right)=g_{i}(x), i=1,2, \ldots, p$, where $g_{i}(x)=2\left[q_{i}\right]_{x}+2 h_{i}$;

(b) $\lim _{n \rightarrow \infty} 2 \pi^{2}(n-1)^{2}\left(x_{n i}^{j_{n i}}-\beta_{n}^{j}\right)=h_{i}(x), i=p+1, p+2, \ldots, m-1$, where $h_{i}(x)=2\left[q_{i}\right]_{x}$; 
(c) $\lim _{n \rightarrow \infty} 2 \pi^{2}(n-1)^{2}\left(x_{n m}^{j_{n i}}-\gamma_{n}^{j}\right)=f_{m}(x)$, where $f_{m}(x)=2\left[q_{m}\right]_{x}$.

Now we can provide a uniqueness theorem and constructive algorithm for the inverse nodal problem on the whole interval $(0,1)$.

Theorem 3.1. Fix $i=1,2, \ldots, m$. Suppose that a set of nodes $X_{i}^{0} \subset X_{i}$ is dense on $(0,1)$. Then the data $X_{i}^{0}$ can uniquely determine $q_{i}(x)$ a.e. on $(0,1)$ and $h_{i}$. And the constructive algorithm is as follows:

(a) $q_{i}(x)=g_{i}^{\prime}(x), h_{i}=g_{i}(0) / 2, i=1,2, \ldots, p$;

(b) $q_{i}(x)=h_{i}^{\prime}(x), i=p+1, p+2, \ldots, m-1$;

(c) $q_{m}(x)=f_{m}^{\prime}(x)$.

4. Inverse nodal problems on the subinterval

Let $0<a<1$. Denote the node $X_{i, a}^{0} \subset X_{i} \cap(a, 1)$ on a subinterval $(a, 1)$ of the edge $e_{i}$. From Theorem 3.1 we get the following statement.

Theorem 4.1. Fix $i=1,2, \ldots, m$ and $a \in(0,1)$. Suppose that a set of nodes $X_{i, a}^{0}$ is dense on $(a, 1)$. Then the data $X_{i, a}^{0}$ can uniquely determine $q_{i}(x)$ a.e. on $(a, 1)$.

For the node set $X_{i, a}^{0}$, set $X_{a}^{0}=\bigcup_{i=1}^{m-1} X_{i, a}^{0}, S_{X_{a}^{0}}=\left\{n: \exists j, i, x_{n i}^{j} \in X_{i, a}^{0}\right\}, S_{X_{m, a}^{0}}=\{n:$ $\left.\exists j, x_{n m}^{j} \in X_{m, a}^{0}\right\}$, and

$$
\Lambda_{1}:=\left\{\lambda_{n 1}\right\}_{n \in S_{X_{a}^{0}}}, \quad \Lambda_{2}:=\left\{\lambda_{n 2}\right\}_{n \in S_{X_{m, a}^{0}}} .
$$

Introduce the counting function

$$
N_{k}(t):=\#\left\{n \mid \lambda_{n k}<t^{2}, \lambda_{n k} \in \Lambda_{k}\right\}, \quad t \in \mathbb{R}^{+}, k=1,2,
$$

and assume that

$$
\Lambda_{1} \cap \Lambda_{2}=\emptyset, \quad \lim _{t \rightarrow \infty} \frac{N_{k}(t)}{t}=: \frac{\alpha_{k}}{\pi}, \quad 0 \leq \alpha_{k} \leq 1 .
$$

Finally, the result that the partial nodes on the subinterval $[a, 1]$ of each edge $e_{i}$ uniquely determine $q_{i}(x)$ on the whole $(0,1)$ and $h_{i}$ is described as follows.

Theorem 4.2. Fix $a \in(0,1)$. Suppose that a set of nodes $X_{i, a}^{0}$ is twin-dense in $(a, 1)$, $i=1,2, \ldots, m$. If there exist $\beta \in[0,1]$ and $\delta>0$ such that for sufficiently large $t$

$$
\sum_{k=1}^{2} N_{k}(t) \geq 2 a\left\{\beta\left[\frac{t}{\pi}+\frac{1}{2}\right]+(1-\beta)\left(\left[\frac{t}{\pi}\right]+\frac{1}{2}\right)+O\left(t^{-\delta}\right)\right\}
$$


holds, where $[x]$ denotes the largest integer not greater than $x$, then $\bigcup_{i=1}^{m} X_{i, a}^{0}$ uniquely determines $q_{i}(x)(i=1,2, \ldots, m)$ a.e. on $[0,1]$ and $h_{i}(i=1,2, \ldots, p)$.

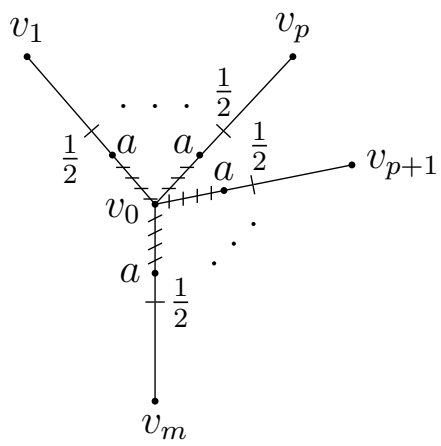

Figure 4.1: Graph with $m$ edges.

Take the node set $X_{i, a}^{0}(i=2,3, \ldots, m)$ such that the eigenvalue sets responding to $S_{X_{i, a}^{0}}$ satisfy $\Lambda_{1}=\left\{\lambda_{n 1}\right\}_{n \geq N_{0}}$ and $\Lambda_{2}=\left\{\lambda_{n 2}\right\}_{n \geq N_{0}}$. Select $\Lambda_{3} \subset\left\{\lambda_{n 3}\right\}_{n \geq 1}$ and $\# \Lambda_{3}=2 N_{0}$. Assume that $\Lambda_{1} \cap \Lambda_{2}=\emptyset$ and $\prod_{j=1}^{p} C_{j}\left(1, \lambda_{n k}\right) \prod_{j=p+1}^{m} S_{j}\left(1, \lambda_{n k}\right) \neq 0$ for all $\lambda_{n k} \in \Lambda_{1} \cup \Lambda_{2}$. From Theorem 4.2 , together with Theorem 3.2 in [1], we have the following corollary.

Corollary 4.3. Fix $a \in(0,1)$. Suppose that a set of nodes $X_{i, a}^{0}$ is twin-dense in $(a, 1)$, $i=2,3, \ldots, m$. Then $\bigcup_{i=2}^{m} X_{i, a}^{0}$ and $\Lambda_{3}$ uniquely determine $q_{i}(x)(i=1,2, \ldots, m)$ a.e. on $[0,1]$ and $h_{i}(i=1,2, \ldots, p)$.

Remark 4.4. (1) In Corollary 4.3 one has $\lim _{t \rightarrow \infty} \frac{N_{k}(t)}{t}=: \frac{1}{\pi}$ so the condition 4.2 is true.

(2) In Theorem 4.2 one needs an assumption that a set of nodes $X_{i, a}^{0}$ is "twin-dense" in $(a, 1)$. In fact, one may use "paired dense" defined in Definition 1.1 in [6] instead of "twin-dense". The conclusion of the theorem still holds.

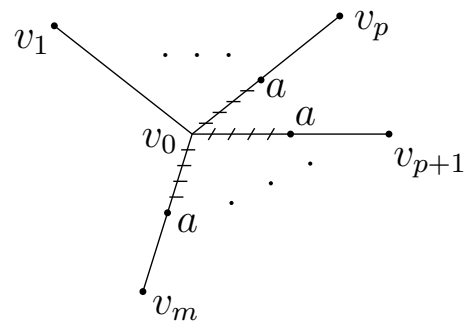

Figure 4.2: Graph with $m$ edges.

Proof of Theorem 4.2. For proving the theorem, together with $L$ we consider a boundary value problem $\widetilde{L}=L(\widetilde{q}, \widetilde{h})$ of the same form but with a different potential $q$ and the 
parameter $h$. We agree that if a certain symbol $\alpha$ denotes an object related to $L$, then $\widetilde{\alpha}$ will denote an analogous object related to $\widetilde{L}$. Consider two boundary value problems $L$ and $\widetilde{L}$ with the assumption that $X_{i, a}^{0}=\widetilde{X}_{i, a}^{0}$.

Step 1: To deduce that $M_{j}\left(a, \lambda_{n k}\right)=\widetilde{M}_{j}\left(a, \lambda_{n k}\right)$ for all $\lambda_{n k} \in \Lambda_{1} \cup \Lambda_{2}$. Under the assumption of Theorem 4.2, from Theorem 4.1 and Lemma 1 in [20] we know

$$
q_{i}(x)=\widetilde{q}_{i}(x) \text { for } x \in[a, 1] \quad \text { and } \quad \lambda_{n k}=\widetilde{\lambda}_{n k} \text { for all } \lambda_{n k} \in \Lambda_{1} \cup \Lambda_{2} \text {. }
$$

From this the following equalities hold

$$
\left(C_{i} \widetilde{C}_{i}^{\prime}-\widetilde{C}_{i} C_{i}^{\prime}\right)\left(a, \lambda_{n k}\right)-\left(C_{i} \widetilde{C}_{i}^{\prime}-\widetilde{C}_{i} C_{i}^{\prime}\right)\left(x_{n k}^{j_{n k}}, \lambda_{n k}\right)=\int_{x_{n k}^{j_{n k}}}^{a}\left(\widetilde{q}_{i}-q_{i}\right)(t)(C \widetilde{C})\left(t, \lambda_{n k}\right) d t \equiv 0
$$

for $i=1,2, \ldots, p$ and

$$
\left(S_{i} \widetilde{S}_{i}^{\prime}-\widetilde{S}_{i} S_{i}^{\prime}\right)\left(a, \lambda_{n k}\right)-\left(S_{i} \widetilde{S}_{i}^{\prime}-\widetilde{S}_{i} S_{i}^{\prime}\right)\left(x_{n k}^{j_{n k}}, \lambda_{n k}\right)=\int_{x_{n k}^{j_{n k}}}^{a}\left(\widetilde{q}_{i}-q_{i}\right)(t)(S \widetilde{S})\left(t, \lambda_{n k}\right) d t \equiv 0
$$

for $i=p+1, p+2, \ldots, m$, where $x_{n k}^{j_{n k}} \in[a, 1]$. Therefore we have

$$
M_{i}\left(a, \lambda_{n k}\right)=\widetilde{M}_{i}\left(a, \lambda_{n k}\right) \quad \text { for all } \lambda_{n k} \in \Lambda_{1} \cup \Lambda_{2}
$$

Step 2: To deduce that $M_{j}(a, \lambda)=\widetilde{M}_{j}(a, \lambda)$ for all $\lambda \in \mathbb{C}$. For $i=1,2, \ldots, p$ let us introduce

$$
\begin{aligned}
H_{i}(a, \lambda) & =C_{i}(a, \lambda) \widetilde{C}_{i}^{\prime}(a, \lambda)-C_{i}^{\prime}(a, \lambda) \widetilde{C}_{i}(a, \lambda) \\
& =C_{i}(a, \lambda) \widetilde{C}_{i}(a, \lambda)\left(M_{i}(a, \lambda)-\widetilde{M}_{i}(a, \lambda)\right) .
\end{aligned}
$$

Together with Step 1, 4.4 shows that

$$
H_{i}\left(a, \lambda_{n k}\right)=0
$$

for all $\lambda_{n k} \in \Lambda_{1} \cup \Lambda_{2}$. From (2.1) and (4.3) we get

$$
\left|H_{i}(a, \lambda)\right|=O\left(\mathrm{e}^{2 a \tau}\right)
$$

for $|\lambda| \rightarrow \infty$. On the other hand, by virtue of (2.1), (2.3) and (4.4), this yields

$$
\left|H_{i}(a, \lambda)\right|=o\left(\mathrm{e}^{2 a \tau}\right)
$$

for $|\lambda| \rightarrow \infty$ in any sector $\varepsilon<\arg \lambda<\pi-\varepsilon$ for some $\varepsilon>0$. Define

$$
F(\lambda):=\frac{H_{i}(a, \lambda)}{\prod_{k=1}^{2} W_{k}(\lambda)}
$$


where

$$
W_{k}(\lambda):=\prod_{\lambda_{n k} \in \Lambda_{1} \cup \Lambda_{2}}\left(1-\frac{\lambda}{\lambda_{n k}}\right), \quad k=1,2 .
$$

If $\lambda_{n k}=0$, we substitute $1-\lambda / \lambda_{n k}$ by $\lambda$. By virtue of 4.5 , we see that $F(\lambda)$ is an entire function in $\lambda$.

Next we will prove $F(\lambda) \equiv 0$. Firstly, since $N_{k}\left(\rho_{n k}\right)=n-1$ as $n \rightarrow \infty$, it follows from (4.1) that

$$
\lim _{n \rightarrow \infty} \frac{n}{\rho_{n k}}=\frac{\alpha_{k}}{\pi}, \quad k=1,2 .
$$

Then Lemma 2.1, together with (4.9), shows that there exist constants $c>0$ and $C>0$ such that

$$
\frac{1}{\left|W_{k}(\lambda)\right|} \leq C \mathrm{e}^{-\alpha_{k} \tau+\varepsilon r}, \quad \forall \lambda \in G_{c}
$$

where $\varepsilon>0$ is arbitrary, and

$$
G_{c}:=\left\{\lambda:\left|\rho-\rho_{n k}\right| \geq \frac{1}{8} c, \lambda_{n k} \in \Lambda_{k}, k=1,2\right\} .
$$

Therefore we obtain

$$
\frac{1}{\left|W_{1}(\lambda) W_{2}(\lambda)\right|} \leq C \mathrm{e}^{-\left(\alpha_{1}+\alpha_{2}\right) \tau+2 \varepsilon r}, \quad \forall \lambda \in G_{c}
$$

for sufficiently large $\lambda$. Moreover, from 4.2 we deduce that $\alpha_{1}+\alpha_{2} \geq 2 a$. Thus 4.6, 4.10) and 4.8 imply

$$
|F(\lambda)|=O\left(\mathrm{e}^{2 \varepsilon r}\right), \quad \forall \lambda \in G_{c}
$$

for sufficiently large $\lambda$. Consequently the maximum modulus principle shows that

$$
|F(\lambda)| \leq C \mathrm{e}^{2 \varepsilon|\lambda|}, \quad \lambda \in \mathbb{C} .
$$

We see that $F(\lambda)$ is of zero exponential type by the arbitrariness of $\varepsilon$. On the other hand, by the known method in [7], we get that for large $|y|$,

$$
\ln \left|W_{1}(i y) W_{2}(i y)\right|=\int_{1}^{\infty} \frac{\sum_{k=1}^{2} N_{k}(\sqrt{t})}{t} \frac{y^{2}}{y^{2}+t^{2}} d t+O(1) .
$$

Combining these with $(4.2)$ and 4.12 yields

$$
\ln \left|W_{1}(i y) W_{2}(i y)\right| \geq 2 a \sqrt{\frac{|y|}{2}}+O(1),
$$

which follows

$$
\left|W_{1}(i y) W_{2}(i y)\right| \geq C e^{2 a \sqrt{\frac{|y|}{2}}}, \quad C>0 \text { is some constant. }
$$


By virtue of (4.7), we get

$$
\left|H_{i}(a, i y)\right|=o\left(e^{2 a \sqrt{\frac{|y|}{2}}}\right)
$$

Hence

$$
|F(i y)|=o(1)
$$

which follows

$$
F(\lambda) \equiv 0, \quad \lambda \in \mathbb{C}
$$

by Lemma 2.2 and 4.11. Consequently, for $i=1,2, \ldots, p$,

$$
H_{i}(a, \lambda) \equiv 0 \quad \text { and } \quad M_{i}(a, \lambda) \equiv \widetilde{M}_{i}(a, \lambda), \quad \lambda \in \mathbb{C},
$$

due to (4.4). Similarly, we also get that for $i=p+1, p+2, \ldots, m$,

$$
M_{i}(a, \lambda) \equiv \widetilde{M}_{i}(a, \lambda), \quad \lambda \in \mathbb{C}
$$

Step 3: To deduce that $q_{i}(x)=\widetilde{q}_{i}(x)(i=1,2, \ldots, m)$ a.e. on $[0,1]$ and $h_{i}=\widetilde{h}_{i}$ $(i=1,2, \ldots, p)$. From the fact that for $i=1,2, \ldots, m$,

$$
M_{i}(a, \lambda) \equiv \widetilde{M}_{i}(a, \lambda), \quad \lambda \in \mathbb{C}
$$

By Borg-Marchenko uniqueness theorem [4], we obtain

$$
q_{i}(x)=\widetilde{q}_{i}(x), \quad i=1,2, \ldots, m \text {, a.e. on }[0,1] \quad \text { and } \quad h_{i}=\widetilde{h}_{i}, \quad i=1,2, \ldots, p \text {. }
$$

The proof is finished.

\section{References}

[1] N. P. Bondarenko, Partial inverse problems for the Sturm-Liouville operator on a star-shaped graph with mixed boundary conditions, J. Inverse Ill-Posed Probl. 26 (2018), no. 1, 1-12.

[2] Y. H. Cheng, Reconstruction of the Sturm-Liouville operator on a p-star graph with nodal data, Rocky Mountain J. Math. 42 (2012), no. 5, 1431-1446.

[3] S. Currie and B. A. Watson, Inverse nodal problems for Sturm-Liouville equations on graphs, Inverse Problems 23 (2007), no. 5, 2029-2040.

[4] F. Gesztesy and B. Simon, Inverse spectral analysis with partial information on the potential II: The case of discrete spectrum, Trans. Amer. Math. Soc. 352 (2000), no. $6,2765-2787$. 
[5] Y. Guo and G. Wei, Inverse problems: Dense nodal subset on an interior subinterval, J. Differential Equations 255 (2013), no. 7, 2002-2017.

[6] _ The sharp conditions of the uniqueness for inverse nodal problems, J. Differential Equations 266 (2019), no. 7, 4432-4449.

[7] M. Horváth, On the inverse spectral theory of Schrödinger and Dirac operators, Trans. Amer. Math. Soc. 353 (2001), no. 10, 4155-4171.

[8] P. Kuchment, Quantum graphs I: Some basic structures, Waves Random Media 14 (2004), no. 1, S107-S128.

[9] C. K. Law, W.-C. Lian and W.-C. Wang, The inverse nodal problem and the Ambarzumyan problem for the p-Laplacian, Proc. Roy. Soc. Edinburgh Sect. A 139 (2009), no. 6, 1261-1273.

[10] C. K. Law and V. Pivovarchik, Characteristic functions of quantum graphs, J. Phys. A 42 (2009), no. 3, 035302, 12 pp.

[11] C. K. Law and C.-F. Yang, Reconstructing the potential function and its derivatives using nodal data, Inverse Problems 14 (1998), no. 2, 299-312.

[12] B. Ya. Levin, Distribution of Zeros of Entire Functions, (Russian) Gosudarstv. Izdat. Tehn.-Teor. Lit., Moscow, 1956.

[13] N. Levinson, Gap and Density Theorems, American Mathematical Society Colloquium Publications 26, American Mathematical Society, New York, 1940.

[14] J. R. McLaughlin, Inverse spectral theory using nodal points as data-A uniqueness result, J. Differential Equations 73 (1988), no. 2, 354-362.

[15] V. Pivovarchik, Inverse problem for the Sturm-Liouville equation on a simple graph, SIAM J. Math. Anal. 32 (2000), no. 4, 801-819.

[16] Yu. V. Pokornyı̆, O. M. Penkin, V. L. Pryadiev, A. V. Borovskikh, K. P. Lazarev and S. A. Shabrov, Differential Equations on Geometric Graphs, Fiziko-Matematicheskaya Literatura, Moscow, 2005.

[17] C.-F. Yang, Inverse spectral problems for the Sturm-Liouville operator on a d-star graph, J. Math. Anal. Appl. 365 (2010), no. 2, 742-749.

[18] C.-F. Yang and X.-P. Yang, Inverse nodal problems for differential pencils on a starshaped graph, Inverse Problems 26 (2010), no. 8, 085008, 15 pp. 
[19] X.-F. Yang, A new inverse nodal problem, J. Differential Equations 169 (2001), no. 2, 633-653.

[20] V. Yurko, Inverse nodal problems for Sturm-Liouville operators on star-type graphs, J. Inverse Ill-Posed Probl. 16 (2008), no. 7, 715-722.

Chuan-Fu Yang and Sheng-Yu Guan

Department of Applied Mathematics, School of Science, Nanjing University of Science and Technology, Nanjing 210094, Jiangsu, China

E-mail addresses: chuanfuyang@njust.edu.cn, guanshengyu@njust.edu.cn 